\title{
A 20-year evolution of cardiac performance in microgravity in a male astronaut
}

\author{
Ulrich Limper ${ }^{1,2}\left(\mathbb{D} \cdot\right.$ Stefan Moestl ${ }^{1} \cdot$ Jens Tank ${ }^{1} \cdot$ Gordon K. Prisk $^{3} \cdot$ Karsten Heusser $^{1} \cdot$ Fabian Hoffmann $^{1,4}$. \\ Axel Goßmann ${ }^{5} \cdot$ Pierre-Francoise Migeotte $^{6} \cdot$ Peter Gauger $^{1} \cdot$ Luis E. J. Beck $^{1} \cdot$ Hans W. Schlegel $^{7}$. \\ Benjamine D. Levine ${ }^{8} \cdot$ Jens Jordan ${ }^{1,9}$
}

Received: 8 November 2019 / Accepted: 9 December 2019

(c) Springer-Verlag GmbH Germany, part of Springer Nature 2020

\section{Dear Editors,}

Today's astronauts are highly selected and physically fit professionals with an average age ranging from 40 to 50 years [5]. Age at the time of astronaut selection has gradually increased over the years [5], and future commercial space tourism will expose older persons with comorbidities to space conditions. Weightlessness elicits an acute cephalad fluid shift; more chronically, cardiac atrophy and altered autonomic cardiovascular control [3] limit the ability of the cardiovascular system to cope with hemodynamic challenges after the astronaut's return from space. With increasing age, the capacities of autonomic cardiovascular adjustments deteriorate [1], possibly resulting in changes in the tolerance to gravitational stress [10] and in physical performance. Increasing age of professional and recreational astronauts could conceivably affect the cardiovascular autonomic response to weightlessness. With the exception of a case report on orthostatic responses in a 77-year-old astronaut [9], studies on cardiovascular aging in astronauts are scarce. We had the unique opportunity to assess the cardiovascular

Electronic supplementary material The online version of this article (https://doi.org/10.1007/s10286-019-00657-1) contains supplementary material, which is available to authorized users.

Ulrich Limper

Ulrich.limper@dlr.de

1 German Aerospace Center (DLR), Institute of Aerospace Medicine, Linder Hoehe, 51147 Cologne, Germany

2 Department of Anesthesiology and Intensive Care Medicine, Merheim Medical Center (CMMC), Witten/Herdecke University, Witten, Germany

3 Departments of Medicine and Radiology, University of California, San Diego, CA, USA

4 Department of Internal Medicine III, University of Cologne, Cologne, Germany responses of an older male astronaut during parabolic flights producing short-term weightlessness. We subsequently compared these responses to measurements obtained for this same astronaut 20 years earlier in space.

In 1993, the male astronaut under study had spent almost 10 days in space as crew member of the second German Spacelab mission D2 aboard STS-55 Space Shuttle Columbia. Twenty years later, at age 62 years, he participated in a parabolic flight campaign. In the intervening years, he had remained physically active and had passed the yearly US National Aeronautics and Space Administration (NASA) medical tests for astronauts. NASA provided D2 mission data, and the analysis was approved by the NASA Institutional Review Board (Protocol number Pro1372). The parabolic flight study was approved by the ethical committee of University Witten/Herdecke. The astronaut provided written informed consent including consent for this publication.

During D2, haemodynamic and cardiovascular autonomic testing were conducted on the astronaut in the supine position 270 days before launch, after 4 days in space, and within the first week after his return to Earth. Testing included cardiac output measurements by the acetylene gas rebreathing method. During the parabolic flights, we measured cardiac output under the $1 \mathrm{G}$ and weightlessness conditions using

5 Department of Radiology, Cologne-Merheim Medical Center (CMMC), Witten/Herdecke University, Witten, Germany

6 Department of Cardiology, Université de Libre de Bruxelles, Brussels, Belgium

7 European Space Agency, European Astronaut Centre, Cologne, Germany

8 Division of Cardiology, Department of Internal Medicine, University of Texas Southwestern Medical Center, Dallas, TX, USA

9 Chair of Aerospace Medicine, University of Cologne, Cologne, Germany 
the inert gas rebreathing method (INNOCOR®; Innovision ApS, Glamsbjerg, Denmark). We evaluated 57 parabolic flight inert gas rebreathing data sets and an identical number of simultaneously collected electrocardiography data sets and finger blood pressure tracings. We obtained measurements supine at $1 \mathrm{G}$ and during supine and upright transitions from $1.8 \mathrm{G}$ to weightlessness. The astronaut received $100 \mu \mathrm{g}$ scopolamine-hydrobromide subcutaneously $30 \mathrm{~min}$ before the parabolic flights to prevent motion sickness.

At the time of the D2 mission, cardiac mass was assessed by magnetic resonance imaging before and after spaceflight [7]. At the time of the parabolic flight campaign, we assessed cardiac structure and function through dynamic cardiovascular magnetic resonance imaging.

At the time of the shuttle mission, the astronauts's body mass index (BMI) was $25 \mathrm{~kg} / \mathrm{m}^{2}$ [8]; 20 years later, it has increased to $29 \mathrm{~kg} / \mathrm{m}^{2}$. Blood pressure and heart rate evolutions at rest and during physical exercise over the years are shown in Fig. 1. Peak oxygen consumption progressively declined over time. Left ventricular mass was $181 \mathrm{~g}$ before and $166 \mathrm{~g}$ after the shuttle mission and had decreased to 123 g 20 years later (Electronic Supplementary Material
[ESM] Table S1). Heart rate varied in the time and frequency domain while supine or during orthostatic stress and changed only modestly with age. Supine low-frequency oscillations of systolic blood pressure were $2 \mathrm{mmHg}^{2}$ during the shuttle mission and $9 \mathrm{mmHg}^{2} 20$ years later (ESM Table S2).

Supine cardiac output was $8.0 \mathrm{~L} \cdot \mathrm{min}^{-1}$ before the shuttle launch and $7.0 \mathrm{~L} \cdot \mathrm{min}^{-1}$ on flight day 4 , and it remained reduced 7 days after the return to Earth. Total peripheral resistance was $822 \mathrm{dyn} \cdot \mathrm{s} \cdot \mathrm{cm}^{-5}$ while supine at $1 \mathrm{G}$ and $1056 \mathrm{dyn} \cdot \mathrm{s} \cdot \mathrm{cm}^{-5}$ in weightlessness (ESM Table S3). Cardiac power output, which is derived from cardiac output and mean arterial pressure, was $1.45 \mathrm{~W}$ before and $1.45 \mathrm{~W}$ during spaceflight; 20 years later, supine cardiac output at $1 \mathrm{G}$ was $3.5 \pm 0.3 \mathrm{~L} \cdot \mathrm{min}^{-1}$ and increased moderately with the transition from $1.8 \mathrm{G}$ to weightlessness to $4.0 \pm 0.5 \mathrm{~L} \cdot \mathrm{min}^{-1}$ $(p=0.010)$. Total peripheral resistance was substantially increased while supine at $1 \mathrm{G}$ and in weightlessness. During the parabolic flight campaign, supine cardiac power output at $1 \mathrm{G}$ was $0.46 \pm 0.07 \mathrm{~W}$ and increased to $0.67 \pm 0.09 \mathrm{~W}$ with the transition from $1.8 \mathrm{G}$ to weightlessness $(p<0.001)$ (ESM Fig. S1). With standing transitions into weightlessness,
Fig. 1 Longitudinal anthropometric and cardiovascular data of exercise stress testing performed on a yearly basis for the astronaut flight medical examination. a Body mass index $(B M I), \mathbf{b}$ heart rate during rest in sitting position (grey graph) and at peak exercise capacity (black graph), c, d systolic (c) and diastolic (d) arterial pressures during rest in sitting position (grey graph) and at peak exercise capacity (black graph), e peak oxygen consumption (VO2 peak)
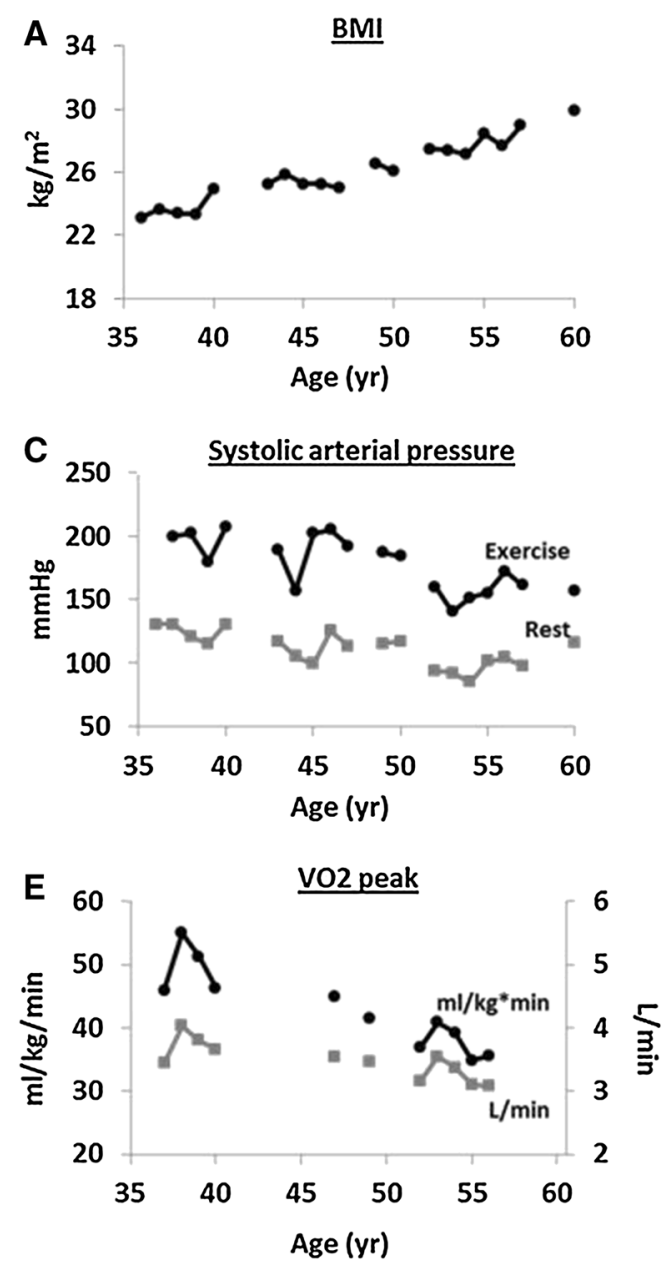
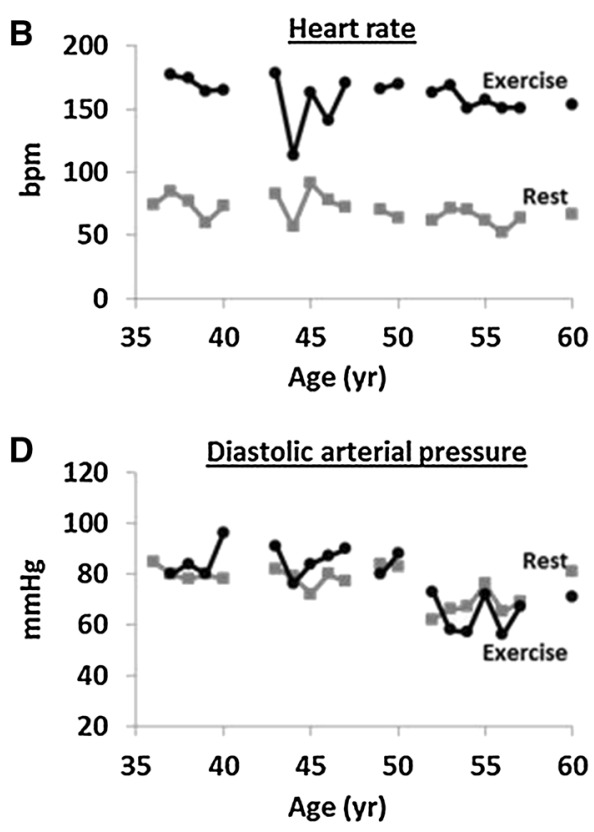
cardiac output increased from $3.2 \pm 0.4$ to $6.0 \pm 0.6 \mathrm{~L} \cdot \mathrm{min}^{-1}$, total peripheral resistance decreased from $1959 \pm 343$ to $1034 \pm 167 \mathrm{dyn} \cdot \mathrm{s} \cdot \mathrm{cm}^{-5}$ and cardiac power output almost doubled (ESM Table S4).

Our findings suggest that aging-associated changes in cardiovascular control mechanisms and in cardiac structure may alter the haemodynamic response to weightlessness. The astronaut under study recapitulated typical aging-associated changes, including increases in pulse pressure, systemic vascular resistance, and blood pressure variability in the low-frequency range, all of which result from sympathetic modulation of vascular tone. Cross-sectional studies applying more direct measurements also showed increased sympathetic support of vascular tone with age [4]. Conversely, cardiac output, cardiac power output, heart rate variability, and baroreflex sensitivity, while still in the physiological range, markedly decreased. Cardiac output tends to decrease after the third decade of life [2]. The combination of intrinsic cardiovascular aging and indirect cardiovascular effects of changes in physical activity and increased adiposity could be causative. Aging-associated changes in cardiovascular structure and autonomic control may have contributed to the profound shifts in cardiac output and power output towards lower levels and total peripheral resistance towards higher levels, both supine at $1 \mathrm{G}$ and during weightlessness.

Our report has important limitations. Observations on a single astronaut cannot be generalized. Furthermore, measurments from real space missions and parabolic flights are difficult to compare; for example, plasma volume tends to decrease over time in space. Low-dose scopolamine application, which has a limited effect on the cardiovascular response to parabolic flights [6], is another limitation. Despite these issues, our findings may have practical implications for an aging professional astronaut population and commercial space tourism, which will not be restricted to highly selected persons. Our preliminary observations, particularly the reduction in cardiac performance, provide an impetus for systematic studies on influences of aging on the cardiovascular response to weightlessness. Preservation of the cardiovascular capacity of aging astronauts is key to enabling human space exploration and future private astronautics.

Acknowledgements This work was supported by the Grants 50WB1255 and 50WB1517 of the German Federal Ministry for Economic Affairs and Energy. We are grateful to the German Aerospace Center for providing the flight opportunity during the 22nd DLR
Parabolic Flight Campaign. We are also thankful to Wolfram Sies for his technical support and to the appointees of NOVESPACE for all their skillful help.

Authors' contribution UL, SM, GKP, AG, PFM, PG, LEJB, HWS contributed substantially to conception and design of the study. UL, SM, GKP, AG, PFM, PG, HWS performed data acquisition. UL, SM, JT, $\mathrm{KH}, \mathrm{FH}, \mathrm{AG}$ performed statistical data analysis. UL, SM, JT, GKP, $\mathrm{KH}, \mathrm{FH}, \mathrm{AG}, \mathrm{PFM}, \mathrm{PG}$, LEJB, HWS, BDL, JJ contributed to interpretation of the data of the work. UL and JJ wrote the first draft of the manuscript. All authors contributed to manuscript revision, read and approved the submitted version.

Data availability The datasets generated during and analyzed during the current study are not publicly available due to protect the medical data of the participant but are available from the corresponding author on reasonable request.

\section{Compliance with ethical standards}

Conflict of interest The authors declare that they have no conflict of interest.

\section{References}

1. Almeida-Santos MA, Barreto-Filho JA, Oliveira JL, Reis FP, da Cunha Oliveira CC, Sousa AC (2016) Aging, heart rate variability and patterns of autonomic regulation of the heart. Arch Gerontol Geriatr 63:1-8

2. Brandfonbrener M, Landowne M, Shock NW (1955) Changes in cardiac output with age. Circulation 12:557-566

3. Eckberg DL, Diedrich A, Cooke WH, et al (2016) Respiratory modulation of human autonomic function: long-term neuroplasticity in space. J Physiol 594:5629-5646

4. Jones PP, Shapiro LF, Keisling GA, et al (2001) Altered autonomic support of arterial blood pressure with age in healthy men. Circulation 104:2424-2429

5. Kovacs GTA, Shadden M (2017) Analysis of age as a factor in NASA astronaut selection and career landmarks. PLoS ONE 12:e0181381

6. Limper U, Gauger P, Beck P, Krainski F, May F, Beck LE (2014) Interactions of the human cardiopulmonary, hormonal and body fluid systems in parabolic flight. Eur J Appl Physiol 114:1281-1295

7. Perhonen MA, Franco F, Lane LD, et al (2001) Cardiac atrophy after bed rest and spaceflight. J Appl Physiol 91:645-653

8. Prisk GK, Verhaeghe S, Padeken D, Hamacher H, Paiva M (2001) Three-dimensional ballistocardiography and respiratory motion in sustained microgravity. Aviat Space Environ Med 72:1067-1074

9. Rossum AC, Ziegler MG, Meck JV (2001) Effect of spaceflight on cardiovascular responses to upright posture in a 77-year-old astronaut. Am J Cardiol 88:1335-1337

10. Shibao C, Grijalva CG, Raj SR, Biaggioni I, Griffin MR (2007) Orthostatic hypotension-related hospitalizations in the United States. Am J Med 120:975-980 